\title{
Study of Epoxy/CNT Nanocomposites Prepared Via Dispersion in the Hardener
}

\author{
Caio Enrico Pizzutto a, Jaqueline Suavea, Jonas Bertholdi, Sérgio Henrique Pezzin ${ }^{\text {a }}$ \\ Luiz Antonio Ferreira Coelho ${ }^{\text {a*, Sandro Campos Amico }}{ }^{\mathrm{b}}$ \\ ${ }^{a}$ Center of Technological Sciences, Santa Catarina State University - UDESC, \\ CEP 89223-100, Joinville, SC, Brazil \\ ' Materials Engineering Departament, Universidade Federal do Rio Grande do Sul - UFRGS, \\ CEP 91501-970, Porto Alegre, RS, Brazil
}

Received: March 15, 2011; Revised: May 11, 2011

\begin{abstract}
In this study, carboxylated (SWCNT-c) and pristine (SWCNT) single-walled carbon nanotubes were randomly dispersed in a hardener prior to mixing it with an epoxy resin. The influence of several parameters on the dispersion process were investigated. The produced samples were characterized by infrared spectroscopy, differential scanning calorimetry, dilatometry, dynamic mechanical analysis, scanning electron microscopy and mechanical testing (tensile, flexural and microhardness). The results obtained with the nanocomposites with SWCNT-c suggested that the lowest time and amplitude of sonication improved the mechanical properties. The use of a solvent (acetone) was important to improve dispersion, ultimately increasing microhardness and Young's Modulus up to 32\%. Nanocomposites with 0.25 wt. (\%) SWCNT-c presented superior mechanical properties compared to those with 0.50 wt. (\%) SWCNT. Two simple mathematical models (rule of mixtures and Halpin-Tsai) were used to predict Young's Modulus of the composites yielding results very close to the experimental ones.
\end{abstract}

Keywords: carbon nanotubes, dispersion, hardener, nanocomposites

\section{Introduction}

In the last decade, much attention has been drawn to carbon nanotubes (CNTs) and their use in a variety of applications. However, major difficulties related to the use of CNTs in polymers remain, including the exfoliation of their original bundled structure, their distribution in polymeric matrices and their homogeneous dispersion using a minimum of 0.5-1.0 wt. (\%) CNT content. These issues are in great part a consequence of the significant attractive forces among carboxylated (SWCNT-c) and pristine (SWCNT) single-walled carbon nanotubes, which are in the order of $0.5 \mathrm{eV}$ (approximately $0.8 \times 10^{-19} \mathrm{~J}$ ) per nanometer throughout the nanotube-to-nanotube contact. This level of energy resembles that of a typical covalent bond ${ }^{1}$.

In general, physical methods, such as sonication, are used to promote CNTs dispersion, even though this may damage their physical structure due to the very high shear stress and shear rate involved, which can reach $70 \mathrm{GPa}$ and $10^{7} \mathrm{~s}^{-1}$, respectively. It should also be pointed out that the nanofillers must have a medium with an appropriate viscosity to allow diffusion ${ }^{1}$. The use of solvents, for instance, is also an alternative to reduce the generally high viscosity of thermoset resins used for nanocomposites.

Another possibility is to initiate the CNTs dispersion in the hardener rather than in the resin. Only few works reported this procedure ${ }^{2-4}$. Gojny and Schulte ${ }^{2}$ verified that the best dispersion was achieved by suspending the nanotubes in the hardener, followed by mild sonication, mixing with the epoxy resin, and an extra sonication step. On the other hand, Chen et al. ${ }^{3}$ compared wear rate measurements of samples with CNTs first dispersed in the hardener or in the resin, by asymmetric centrifugation, and found that they were not significantly different. Furthermore, the use of surfactants or solvents to aid this process is still an open issue. A previous study ${ }^{4}$ showed that significant increase in some mechanical properties is achieved by dispersing carboxylated SWCNTs in the hardener, indicating this to be a possible route for preparing nanocomposites.

The other major issue related to nanocomposites regards the need for good interfacial adhesion between nanotubes and the polymer matrix in order to allow efficient stress transfer between them, which is strongly dependent on the type of CNTs and the nature of the polymer matrix. On this context, functionalization of the nanotube surface and the use of surfactants are believed to enhance interfacial adhesion $^{5}$, even though this process may also damage the surface of the carbon nanotube ${ }^{6}$.

An additional benefit associated with functionalization is that it may also aid dispersion due to a strong interaction with the dispersion medium ${ }^{2,7}$. Although covalent functionalisation of CNTs may be helpful for mechanical reinforcement, it can be deleterious for electrical conductivity. It is possible that functionalization can even hinder the dispersion, specially if the nanotubes become too reactive. Nevertheless, CNTs are able to increase, for instance, the electrical conductivity of an epoxy resin due to their high tendency to form conducting networks as well as lower percolation thresholds. However, impurities, entanglement/dispersion degree and CNT diameter and length complicate the transfer of their good electrical and mechanical properties to the nanocomposites ${ }^{8}$.

In this work, two types of CNTs were dispersed in the hardener, using sonication, a solvent and/or a surfactant, and different stirring techniques for mixing the hardener with the epoxy resin. The effect of these process variants on the properties of CNTs/epoxy nanocomposites was investigated. Besides, two simple micromechanical models to estimate Young's Modulus of the nanocomposites were employed to compare theoretical and experimental results. 


\section{Materials and Methods}

SWCNT-c and SWCNT used in this work were supplied by the Nanomaterials Laboratory at the Federal University of Minas Gerais (UFMG-Brazil) and were produced by electrical arc discharge with 92\% purity (supplier information). The bifunctional diglycidyl ether of bisphenol A (DGEBA) epoxy resin was obtained from Huntsman as Araldite GY251, together with the hardener Aradur HY956. The solvent used was analytical grade acetone, from Cinética Química/ Brazil and, the surfactant, Triton X-100 supplied by Vetec Química Fina/Brazil.

Figure 1 shows a generic route used for preparing all nanocomposites studied in this work. For sonication, a Sonics Vibration 750 equipment was employed. Previous studies published by the group on sonication conditions (i.e. power and time) may be found elsewhere ${ }^{4,9}$ and the final evolution of the solvent was followed via mass balance. The samples were named as shown in Table 1, considering the various parameters studied. For comparison purposes, neat epoxy samples were also prepared using the same curing conditions.

\subsection{Characterization}

Mechanical tests were performed according to ASTM D638M using an EMIC DL30000 universal testing machine with a $1000 \mathrm{kgf}$ load cell operating at a cross-head speed of $5 \mathrm{~mm} / \mathrm{min}$. Flexural properties were determined according to ASTM D790 in the same equipment, with a $100 \mathrm{kgf}$ load cell operating at $2.6 \mathrm{~mm} / \mathrm{min}$. Vickers hardness was measured using $25 \mathrm{~g}$ load and 10 seconds loading time in a Shimadzu HMV-2T equipment. Surface morphology of the tensile fractured specimens was observed under a Zeiss DSM 940A scanning electron microscope (SEM) operating at $10 \mathrm{kV}$. The samples were previously gold-coated using a sputtering device.

The various nanocomposite specimens were also analyzed in a Netzsch $402 \mathrm{C}$ dilatometer. The samples were first heated from room temperature to $150{ }^{\circ} \mathrm{C}$ (at $10{ }^{\circ} \mathrm{C} / \mathrm{min}$ and under $\mathrm{N}_{2}$ atmosphere), followed by a fast cool down to room temperature and a new heating (second run) under the same conditions. Dynamic-mechanical analysis (DMA) on dual cantilever mode was carried out from room temperature to $150^{\circ} \mathrm{C}$ at 5 minutes in a TA Instruments (model 2980) equipment. All measurements were conducted under $0.01 \%$ strain and $1 \mathrm{~Hz}$ frequency. DSC was conducted in a DSC2010 TA Instruments equipment at a heating rate of $10{ }^{\circ} \mathrm{C} / \mathrm{min}$, using a heating/cooling/ heating cycle. Fourier transform infrared transmission spectroscopy (FTIR) analysis, in the $400-3500 \mathrm{~cm}^{-1}$ region, was carried out in a Perkin-Elmer equipment using the $\mathrm{KBr}$ pellet technique.

Raman spectroscopy was employed to characterize specific bands of the SWCNT-c, between 800 and $3400 \mathrm{~cm}^{-1}$, on a Labram Jobim Yvon Micro Raman Spectrometer (wavelength: $632.8 \mathrm{~nm}$ ). In addition, Fourier transform infrared (FTIR) transmission spectra were collected with a Nexus microscope (magnification 10×, probed

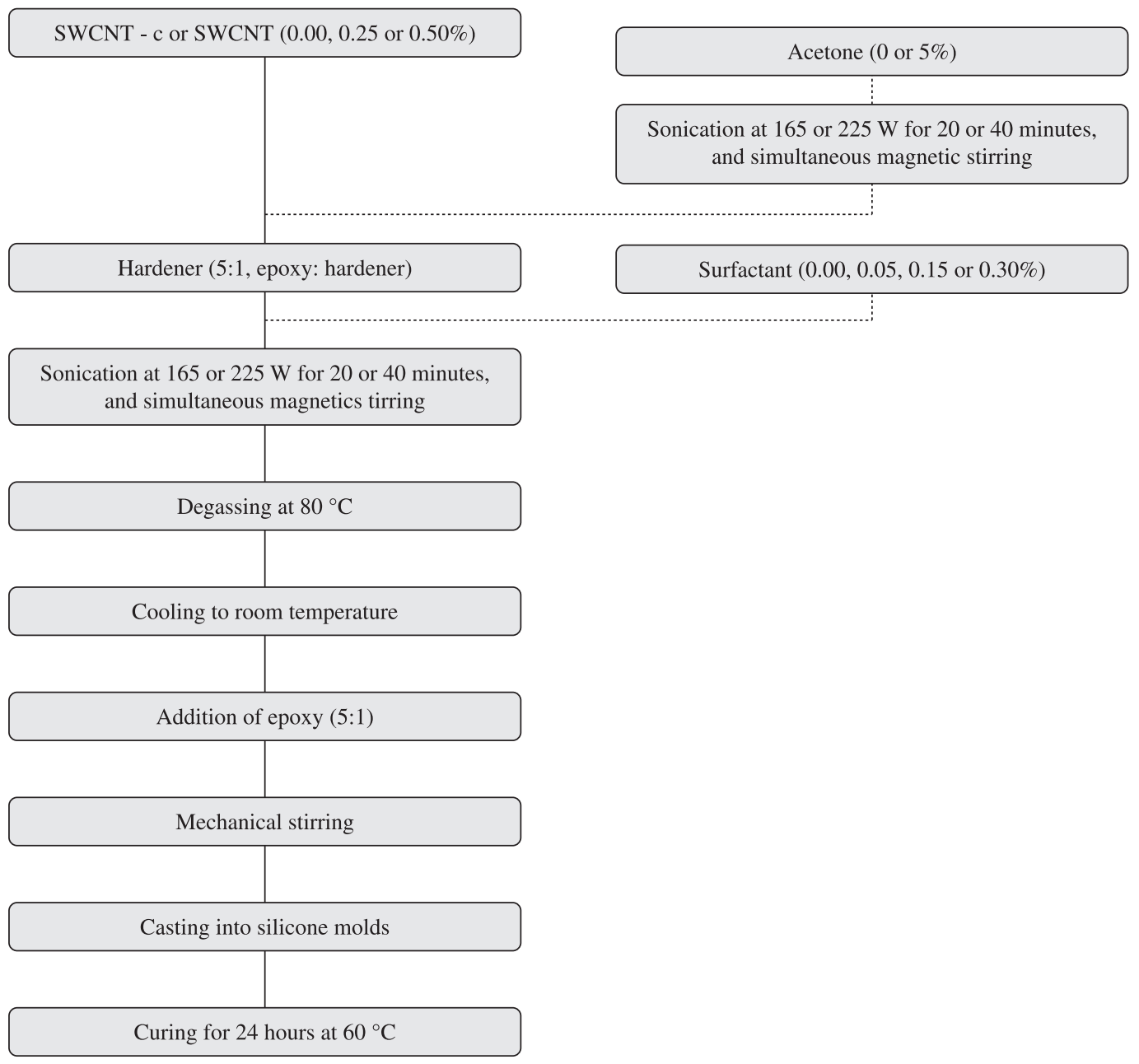

Figure 1. Route used in this work for the preparation of the various nanocomposites. 
Table 1. Nomenclature used for all samples prepared in this work.

\begin{tabular}{|c|c|c|c|c|c|c|}
\hline Sample & $\begin{array}{c}\text { CNT content } \\
(\%)\end{array}$ & $\begin{array}{c}\text { Sonication } \\
\text { period } \\
\text { (minutes) }\end{array}$ & $\begin{array}{c}\text { Sonication } \\
\text { power } \\
(\mathrm{W})\end{array}$ & Solvent used & $\begin{array}{c}\text { Amount of } \\
\text { surfactant } \\
(\%)\end{array}$ & $\begin{array}{c}\text { Degassing at } \\
80^{\circ} \mathrm{C}\end{array}$ \\
\hline SWCNT-c_C25_T20_A22_Man & 0.25 & 20 & 165 & --- & --- & --- \\
\hline SWCNT-c_C25_T20_A22_Mec & 0.25 & 20 & 165 & --- & --- & --- \\
\hline SWCNT-c_C25_T20_A22_Mec_Ace & 0.25 & 20 & 165 & Acetone & --- & Yes \\
\hline SWCNT-c_C25_T40_A30_Mec & 0.25 & 40 & 225 & --- & --- & --- \\
\hline SWCNT-c_C25_T40_A30_Mec_Ace & 0.25 & 40 & 225 & Acetone & --- & Yes \\
\hline SWCNT_C50_T40_A30_Mec & 0.50 & 40 & 225 & --- & --- & --- \\
\hline SWCNT_C50_T40_A30_Mec_Ace & 0.50 & 40 & 225 & Acetone & --- & Yes \\
\hline SWCNT_C50_T40_A30_Mec_Ace_S0.15 & 0.50 & 40 & 225 & Acetone & 0.15 & Yes \\
\hline SWCNT_C50_T40_A30_Mec_Ace_S0.30 & 0.50 & 40 & 225 & Acetone & 0.30 & Yes \\
\hline
\end{tabular}

Man: Manual stirring; Mec: Mechanical stirring at $2000 \mathrm{rpm}$.

region $150 \times 150 \mathrm{~mm}^{2}$ ) attached to a Nicolet spectrometer, in the mid-infrared region $\left(650-3500 \mathrm{~cm}^{-1}\right)$. The measurements were performed under nitrogen purge, with $4 \mathrm{~cm}^{-1}$ of spectral resolution, by averaging 64 scans.

\section{Results and Discussion}

\subsection{Physical and thermal properties}

Table 2 presents the glass transition temperature $\left(\mathrm{T}_{\mathrm{g}}\right)$ determined during the second heating of the DSC analyses of the nanocomposites. $\mathrm{T}_{\mathrm{g}}$ appears independent on the nanotube content and the processing parameters for the samples with carboxylated CNT. In general, no significant change in $\mathrm{T}_{\mathrm{g}}$ was observed, except when surfactant was used, even though no clear trend could be identified since the addition of $0.30 \mathrm{wt}$. (\%) Triton X-100 increased $\mathrm{T}_{\mathrm{g}}$, but lower surfactant content $(0.15$ wt. $(\%))$ decreased $\mathrm{T}_{\mathrm{g}}$ maintaining all other processing parameters unchanged. This may suggest a critical micelle concentration within $0.15-0.30$ wt. (\%) Triton X-100 for 0.50 wt. (\%) SWCNT nanocomposites. The rather small variation in $\mathrm{T}_{g}$ of the nanocomposites prepared using acetone suggests that nearly all solvent was removed, as indicated by the mass balance conducted during the experiments. Indeed, according to Moniruzzaman ${ }^{10}$, complete solvent removal before curing is expected to yield similar cross-linking degree in neat epoxy or epoxy nanocomposites, leading to similar glass transition temperatures.

FTIR was used to monitor the presence of the epoxide group indicated by the presence of an absorption band in the $910-920 \mathrm{~cm}^{-1}$ range, related to the contraction of the $\mathrm{C}-\mathrm{C}$ bond and the stretching of both $\mathrm{C}-\mathrm{O}$ bonds of the epoxy ring ${ }^{11}$. As can be seen for all samples shown in Figure 2, this peak has almost disappeared after curing, which is an evidence of a high degree of crosslinking in the nanocomposites and in the neat epoxy. Similar results may be found in the literature ${ }^{12}$.

In Figure 3, the Raman spectrum of SWCNT-c is presented and two peaks around 1570 and $1350 \mathrm{~cm}^{-1}$ can be seen, referring to the $\mathrm{G}$ band and the disorder-induced $\mathrm{D}$ band, respectively. The intensity of the $\mathrm{D}$ band compared with the $\mathrm{G}$ band indicates high content of disordered structures and defects ${ }^{13}$. The Raman spectra of the noncarboxylated SWCNT was presented elsewhere ${ }^{14}$, showing a very low intensity D band. The high intensity of the D band presented here is expected since covalent functionalization with a carboxylic group changes the physical and chemical structure of SWCNT ${ }^{15}$.

In Figure 4, FTIR spectra of the SWCNTs before and after the oxidation process are presented. It should be noted that the spectrum of the pristine SWCNT is quite similar to others presented in the
Table 2. Glass transition temperature and storage modulus (at $40^{\circ} \mathrm{C}$ ) of the SWCNT and SWCNT-c/epoxy nanocomposites.

\begin{tabular}{lcc}
\hline \multicolumn{1}{c}{ Sample } & $\begin{array}{c}\mathrm{T} \\
\left({ }^{\circ} \mathrm{C}\right)\end{array}$ & $\begin{array}{c}\text { Storage } \\
\text { modulus } \\
(\mathrm{GPa})\end{array}$ \\
\hline Neat Epoxy_Mec & 107 & 2.0 \\
SWCNT-c_C25_T20_A22_Man & 106 & 2.5 \\
SWCNT-c_C25_T20_A22_Mec & 107 & 2.3 \\
SWCNT-c_C25_T20_A22_Mec_Ace & 107 & 2.1 \\
SWCNT-c_C25_T40_A30_Mec & 107 & 2.2 \\
SWCNT-c_C25_T40_A30_Mec_Ace & 106 & 2.2 \\
SWCNT_C50_T40_A30_Mec & 107 & 2.4 \\
SWCNT_C50_T40_A30_Mec_Ace & 110 & 2.5 \\
SWCNT_C50_T40_A30_Mec_Ace_S0.15 & 101 & 2.3 \\
SWCNT_C50_T40_A30_Mec_Ace_S0.30 & 115 & 2.4 \\
\hline
\end{tabular}

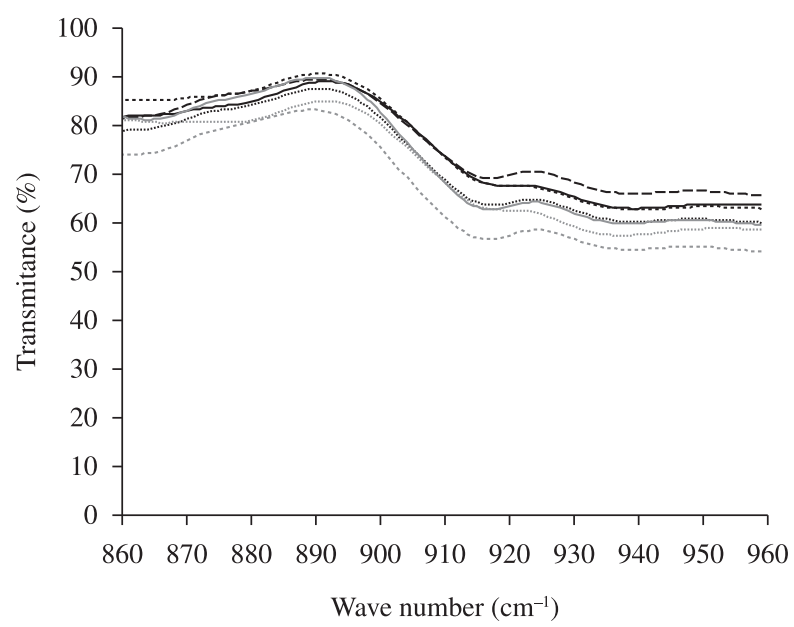

\begin{tabular}{|l|}
\hline - Neat epoxy_man \\
…… SWCNT-c_C25_T40_A30_mec_sol \\
$\cdots-$... SWCNT-c_C10_T40_A30_man \\
--- SWCNT-c_C25_T20_A22_mec_sol \\
- SWCNT-c_C25_T20_A22_mec \\
SW.... SWCNT-c_C25_T40_A30_mec \\
…- Neat epoxy_mec
\end{tabular}

Figure 2. FTIR spectra of SWCNT/epoxy nanocomposites prepared under different conditions. 
literature ${ }^{16,17}$. The presence of typical $\mathrm{C}=\mathrm{O}$ and $\mathrm{O}-\mathrm{H}$ bonds are due to the formation of $\mathrm{COOH}$ groups on the nanotubes after acid treatment. This is evident in the IR spectrum shown in Figure 4. The very broad shoulder peak at $3100-3600 \mathrm{~cm}^{-1}$ is assigned to the $\mathrm{O}-\mathrm{H}$ stretches of terminal carboxyl groups, the peak at $2918 \mathrm{~cm}^{-1}$ can be assigned to the $\mathrm{C}-\mathrm{H}$ stretch, and the peaks near $1730 \mathrm{~cm}^{-1}$ correspond to the carboxylic $\mathrm{C}=\mathrm{O}$ stretching vibrations. The peak at $1585 \mathrm{~cm}^{-1}$ is attributed to the $-\mathrm{C}=\mathrm{C}$ - stretching mode of the $\mathrm{SWCNTs}{ }^{17}$.

The dilatometric behavior of some of the nanocomposites studied in this work is presented in Figure 5. All nanocomposites presented higher dilation than neat epoxy. Besides, higher linear dilation was associated with mild sonication parameters (20 minutes and $165 \mathrm{~W}$ ). According to Gonnet ${ }^{18}$, carbon nanotubes have a negative coefficient of thermal expansion due to the $\mathrm{sp}^{2}$ network and their nanocomposites should present thermal dilation smaller than neat epoxy even at very low CNT content, but this was not confirmed by the results of this work.

\subsection{Mechanical properties}

Figures $6 \mathrm{a}$ and $6 \mathrm{~b}$ present DMA curves of storage modulus versus temperature for the nanocomposites. The storage modulus was found to increase with the addition of CNTs in the range of interest of the epoxy nanocomposites. The highest storage modulus at $40{ }^{\circ} \mathrm{C}$ (see Table 2) was found for the $0.25 \mathrm{wt}$. (\%) SWCNT nanocomposite prepared using acetone and harsh sonication (SWCNT-c_C25_ T40_A30_Ace). However, similarly high values were observed for the nanocomposite with higher SWCNT loading (0.50 wt. (\%)) prepared without solvent and under the same sonication conditions (SWCNT_C50_T40_A30). This suggests that the use of the solvent was effective in improving storage modulus since higher values were found with lower nanofiller content.

In general, the addition of CNTs, either SWCNT or SWCNT-c, was responsible for an increase in storage modulus, and the highest values were found for SWCNT-c_C25_T20_A22_Man and SWCNT_ C50_T40_A30_Mec_Ace. Addition of surfactant also yielded an increase in storage modulus for longer sonication periods. Liao ${ }^{19}$ observed that the use of acetone was important for CNTs debundling in epoxy matrices, increasing in up to $50 \%$ the storage modulus of the matrix. However, in this work, the most significant increase for the nanocomposites prepared with the aid of acetone was $25 \%$.

Table 3 presents the Vickers microhardness values obtained for all samples. These results suggest an increase in hardness with the use of solvent for the carboxylated SWCNT. For the nanocomposites with SWCNT-c, an increase of up to $31 \%$ was observed in comparison with neat epoxy. Suave ${ }^{9}$ studied CNTs dispersion in the resin using tetrahydrofuran (THF) as solvent and observed a small negative variation in this property (ca. $7 \%$ for $0.25 \mathrm{wt}$. (\%) SWCNT-c), whereas $\mathrm{Lau}^{20}$ found an increase of around $20 \%$ (when compared to neat epoxy), reaching $13 \mathrm{HV}$ when using $2 \mathrm{wt}$. (\%) SWCNT prepared using ethanol. It should be noted that the hardness values obtained in this work are much higher than those reported for similar CNTs/ epoxy systems.

Table 3 also presents the tensile properties of the nanocomposites. All samples, except SWCNT_C50_T40_A30_Mec, presented higher Young's modulus than neat epoxy. The more pronounced increase in this property was obtained for the composite with $0.25 \mathrm{wt}$. (\%) SWCNT-c prepared under harsh sonication conditions (40 minutes at $225 \mathrm{~W}$ ) and using the solvent. Surprisingly, this same sample presented a significant decrease in tensile strength. Regarding tensile strength, the highest value was found for carboxylated nanotubes (0.25 wt. (\%)) dispersed under mild sonication conditions (20 minutes at $165 \mathrm{~W}$ ) and using acetone but, even in this case, the increase was not significant considering the standard deviation and therefore it was

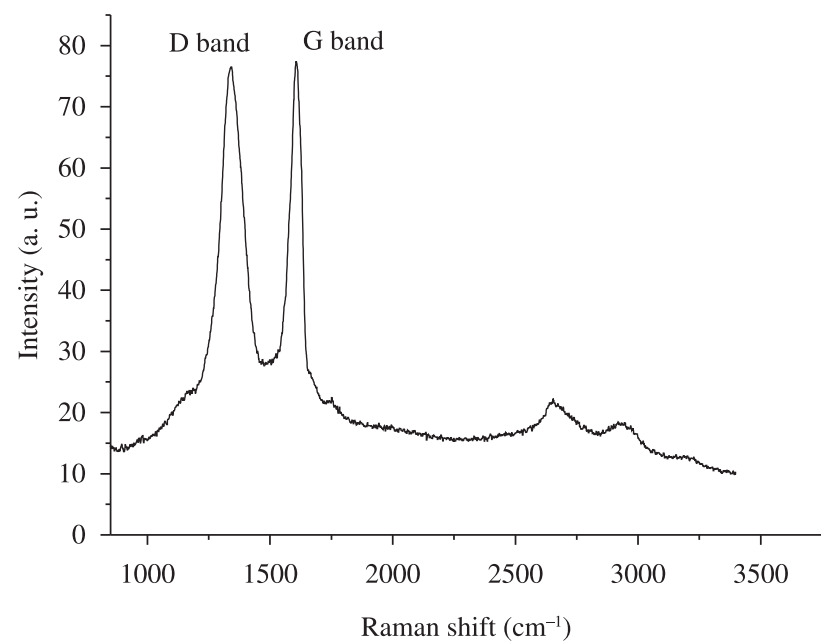

Figure 3. Raman spectra of SWCNT-c.

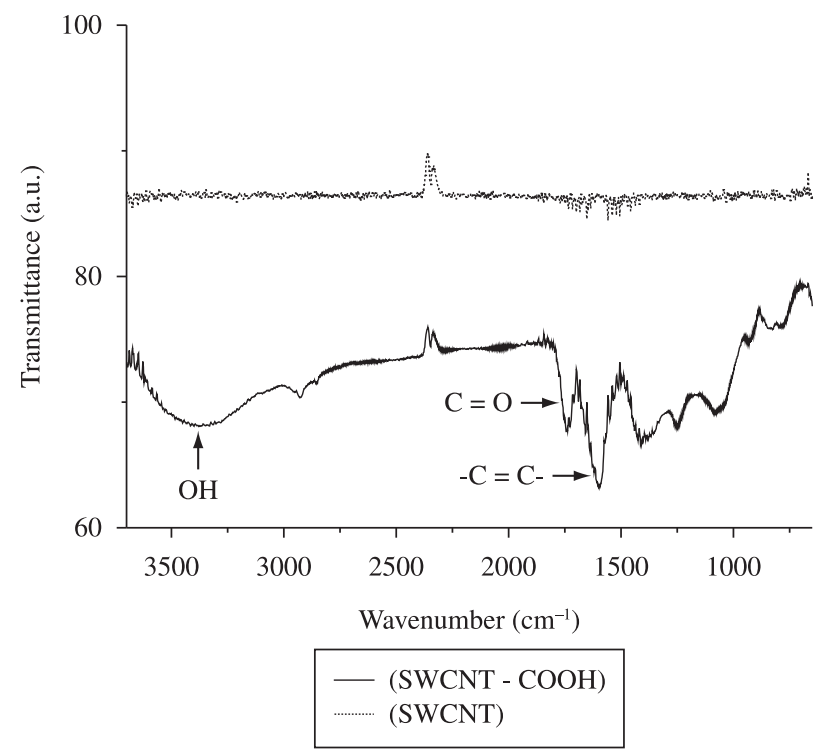

Figure 4. FTIR of pristine SWCNT and SWCNT-c.

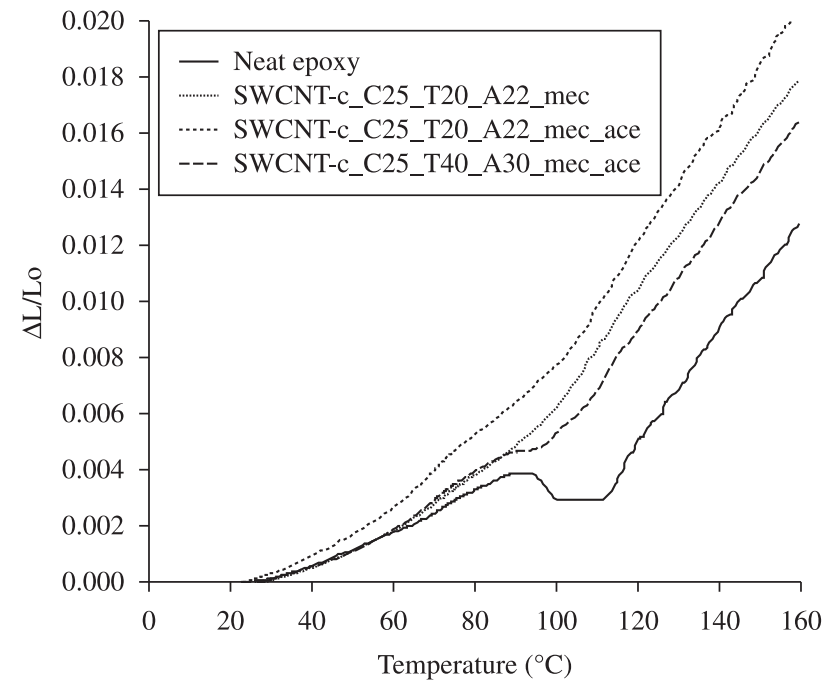

Figure 5. Dilatometric behavior of some SWCNT-c/epoxy nanocomposites (second heating). 


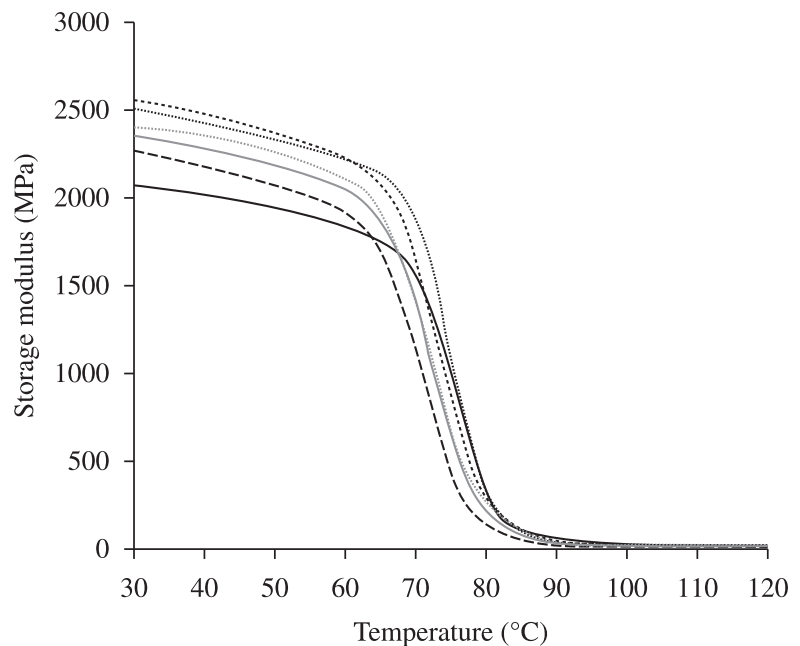

$$
\begin{aligned}
& \text { - Neat epoxy_mec } \\
& \text { ……… SWCNT_C50_T40_A30 } \\
& \text { ….. SWCNT_C25_T40_A30_ace } \\
& --- \text { SWCNT_C50_T20_A22_ace_S0.05 } \\
& \text { - SWCNT_C50_T40_A30_ace_S0.15 } \\
& \text { ……. SWCNT_C50_T40_A30_ace_S0.30 }
\end{aligned}
$$

(a)

\begin{tabular}{|c|c|c|c|c|}
\hline Sample & $\begin{array}{c}\text { Hardness } \\
\text { (HV) }\end{array}$ & $\begin{array}{l}\text { Young's modulus } \\
(\mathrm{GPa})\end{array}$ & $\begin{array}{c}\text { Tensile strength } \\
(\mathrm{MPa})\end{array}$ & $\begin{array}{c}\text { Elongation at } \\
\text { break }(\%)\end{array}$ \\
\hline Neat Epoxy* Man or Mec & $19 \pm 1$ & $3.5 \pm 0.4$ & $39.7 \pm 4.8$ & $1.2 \pm 0.2$ \\
\hline SWCNT-c_C25_T20_A22_Man & $16 \pm 1$ & $4.1 \pm 0.3$ & $28.3 \pm 4.7$ & $0.7 \pm 0.1$ \\
\hline SWCNT-c_C25_T20_A22_Mec & $21 \pm 1$ & $3.5 \pm 0.6$ & $33.0 \pm 11.1$ & $1.1 \pm 0.5$ \\
\hline SWCNT-c_C25_T20_A22_Mec_Ace & $25 \pm 1$ & $4.2 \pm 1.2$ & $45.1 \pm 7.6$ & $1.2 \pm 0.6$ \\
\hline SWCNT-c_C25_T40_A30_Mec & $19 \pm 1$ & $3.7 \pm 0.7$ & $30.0 \pm 6.5$ & $0.9 \pm 0.3$ \\
\hline SWCNT-c_C25_T40_A30_Mec_Ace & $23 \pm 2$ & $4.6 \pm 2.3$ & $19.8 \pm 5.4$ & $0.5 \pm 0.3$ \\
\hline SWCNT_C50_T40_A30_Mec & $24 \pm 3$ & $3.3 \pm 0.2$ & $38.4 \pm 10.9$ & $1.1 \pm 0.3$ \\
\hline SWCNT_C50_T40_A30_Mec_Ace & $20 \pm 1$ & $4.3 \pm 1.0$ & $41.8 \pm 9.1$ & $0.9 \pm 0.2$ \\
\hline SWCNT_C50_T40_A30_Mec_Ace_S0.15 & $19 \pm 1$ & $4.0 \pm 0.9$ & $42.8 \pm 9.8$ & $1.2 \pm 0.3$ \\
\hline SWCNT_C50_T40_A30_Mec_Ace_S0.30 & $16 \pm 1$ & $3.7 \pm 1.1$ & $36.3 \pm 10.1$ & $1.2 \pm 0.5$ \\
\hline
\end{tabular}

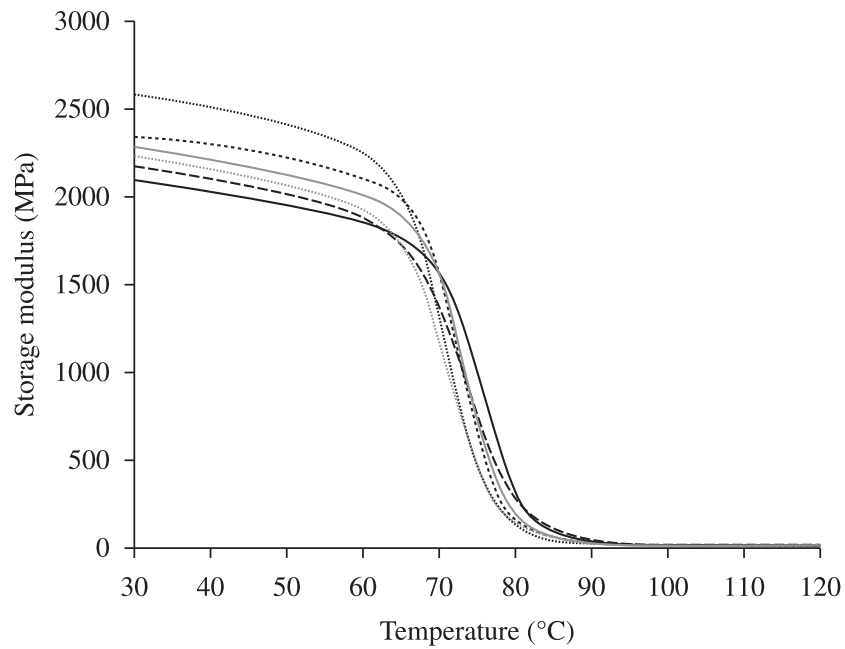

$$
\begin{aligned}
& \text { - Neat epoxy_mec } \\
& \cdots . . . . . . . \\
& \text {...... SWCNT-c_C25_T20_A22_man } \\
& -- \text { SWCNT-c_C25_T20_A22_mec } \\
& \text { - SWCNT-c_C25_T20_A22_mec_ace } \\
& \text { SWCNT-c_C25_T40_A30_mec } \\
& \text { SWCNT-c_C25_T40_A30_mec_ace }
\end{aligned}
$$

(b)

Figure 6. Storage modulus for nanocomposites with SWCNT (a) and SWCNT-c (b).

Table 3. Vickers microhardness and tensile properties of the SWCNT and SWCNT-c/epoxy nanocomposites.

*For the neat epoxy, 30 specimens were tested $(\mathrm{N}=30)$, in the other cases, $\mathrm{N}=8-10$.

not possible to find a clear correlation between processing parameters and tensile strength.

Nevertheless, the use of the solvent shows a clear trend in helping increasing Young's modulus for both SWCNT and SWCNT-c, differently from what was found from observation of the microhardness results. This may have occurred since microhardness (which is not a material property) measurement is much more sensitive to local heterogeneities which may have affected some of the experimental readings.

The results of the flexural tests performed on the nanocomposites prepared with SWCNT-c are summarized in Table 4. No significant variation in flexural modulus was observed. On the other hand, flexural strength showed, in general, a significant improvement when compared to neat epoxy, especially for the $0.25 \mathrm{wt}$. (\%) SWCNT sample sonicated under mild conditions and with manual mixing of the hardener with the epoxy, which presented an increase of $28 \%$. In a similar study by $\mathrm{Chen}^{3}$, modulus and strength were found to decrease when compared to neat epoxy, which was credited to a non-uniform dispersion of CNTs. By adding 2 wt. (\%) SWCNT to epoxy, $\mathrm{Lau}^{20}$ found a decrease of $33 \%$ in flexural strength, which was explained by the presence of aggregates in the nanocomposites.

Scanning electron micrographs of the surface of a tensile fractured nanocomposite obtained in this work are presented in Figure 7. The specimens in general exhibited a relatively smooth surface which is indicative of a brittle fracture. No evidence of poor dispersion was found in Figure 7a and the pull-out of carboxylated SWCNTs taking place at the fractured surface shown in Figure $7 \mathrm{~b}$ indicates poor adhesion between nanotubes and epoxy. The pull-out phenomenon ${ }^{21}$ captured in this SEM image for nanocomposites with CNTs is scarcely seen in the literature.

\subsection{Micromechanical modeling}

A rough estimate of the Young's modulus of a polymeric (nano) composite with randomly oriented CNTs may be theoretically found using the rule of mixtures (Equation 1): 
Table 4. Flexural properties of the SWCNT-c/epoxy nanocomposites.

\begin{tabular}{lll}
\hline \multicolumn{1}{c}{ Sample } & $\begin{array}{c}\text { Flexural } \\
\text { modulus } \\
(\mathrm{GPa})\end{array}$ & $\begin{array}{c}\text { Flexural } \\
\text { strength } \\
(\mathrm{MPa})\end{array}$ \\
\hline Neat Epoxy Man or Mec & $2.8 \pm 0.3$ & $70.4 \pm 5.7$ \\
SWCNT-c_C25_T20_A22_Man & $2.6 \pm 0.2$ & $90.2 \pm 12.6$ \\
SWCNT-c_C25_T20_A22_Mec & $2.8 \pm 0.3$ & $85.5 \pm 19.1$ \\
SWCNT-c_C25_T40_A30_Mec & $2.6 \pm 0.3$ & $70.0 \pm 9.8$ \\
SWCNT-c_C25_T40_A30_Mec_Ace & $2.5 \pm 0.2$ & $73.2 \pm 19.6$ \\
\hline
\end{tabular}

$$
E_{c}=\alpha \beta E_{f} V_{f}+\left(1-V_{f}\right) E_{m}
$$

where: $E$ is the Young's modulus, and subscripts $c, f$ and $m$ refer to the nanocomposite, the nanofiller (in this case, CNTs) and the matrix, respectively, and $V_{f}$ is the CNTs volume fraction in the nanocomposite. The $\beta$ parameter is equal to 0.2 when the CNTs are considered to be arranged in a 3-D random orientation or equal to 0.35 for in-plane (2-D) random orientation. The $\alpha$ parameter may be found using Equation 2:

$$
\alpha=1-\left[\frac{\tanh \left(\alpha \frac{L}{D}\right)}{\alpha \frac{L}{D}}\right]
$$

where: $\alpha=\sqrt{\frac{-3 E_{m}}{2 E_{f} \ln V_{f}}}, L$ e $D$ are the mean length and diameter of the CNTs, respectively.

The Halpin-Tsai theoretical model, given by Equation 3, may also be used to estimate $E_{c}$. The Young's modulus and the aspect ratio $(L / D)$ of the nanotubes may be considered to vary within 500-1000 GPa and 500-1000, respectively. More details about these equations and the values used here can be found in Coleman ${ }^{22}$ and Gojny ${ }^{23}$.

$$
\frac{E_{C}}{E_{m}}=\frac{3}{8}\left[\frac{1+\frac{2 L}{D} \eta_{L} V_{f}}{1-\eta_{L} V_{f}}\right]+\frac{5}{8}\left[\frac{1+2 \eta_{T} V_{f}}{1-\eta_{T} V_{f}}\right]
$$

where: $\eta_{L}=\frac{\frac{E_{f}}{E_{m}}-1}{\frac{E_{f}}{E_{m}}+\frac{2 L}{D}}$ and $\eta_{T}=\frac{\frac{E_{f}}{E_{m}}-1}{\frac{E_{f}}{E_{m}}+2}$.

Using $3.5 \mathrm{GPa}$ as the Young's modulus of the unreinforced polymeric matrix (i.e. neat epoxy) and applying these equations for 0.10-0.50 wt. (\%) CNTs yields the results shown in Table 5. Thus, the experimental results found in this work and in the literature ${ }^{4,22}$ for SWCNTs, MWCNTs and SWCNTs-c with epoxy fitted the estimated range of values found for up to $0.50 \mathrm{wt}$. (\%) CNTs. For detailing purposes, the SWCNT-c_C25_T20_A22_Mec_Ace sample (experimental Young's modulus of $4.2 \mathrm{GPa}$ ) was chosen for comparison. The theoretical estimation of Young's modulus for 0.25 wt. (\%) nanofillers varied within 3.7-4.4 GPa, thus the agreement between experimental and calculated values was very good, and perhaps an intermediate arrangement between pure 2-D and pure 3-D orientation could be expected. Therefore, the obtained experimental results were quite close to the theoretical results obtained using two very simple models. According to the literature, epoxy and other nanocomposites systems $\mathrm{s}^{4,24}$ may yield results just as accurate as more sophisticated models.
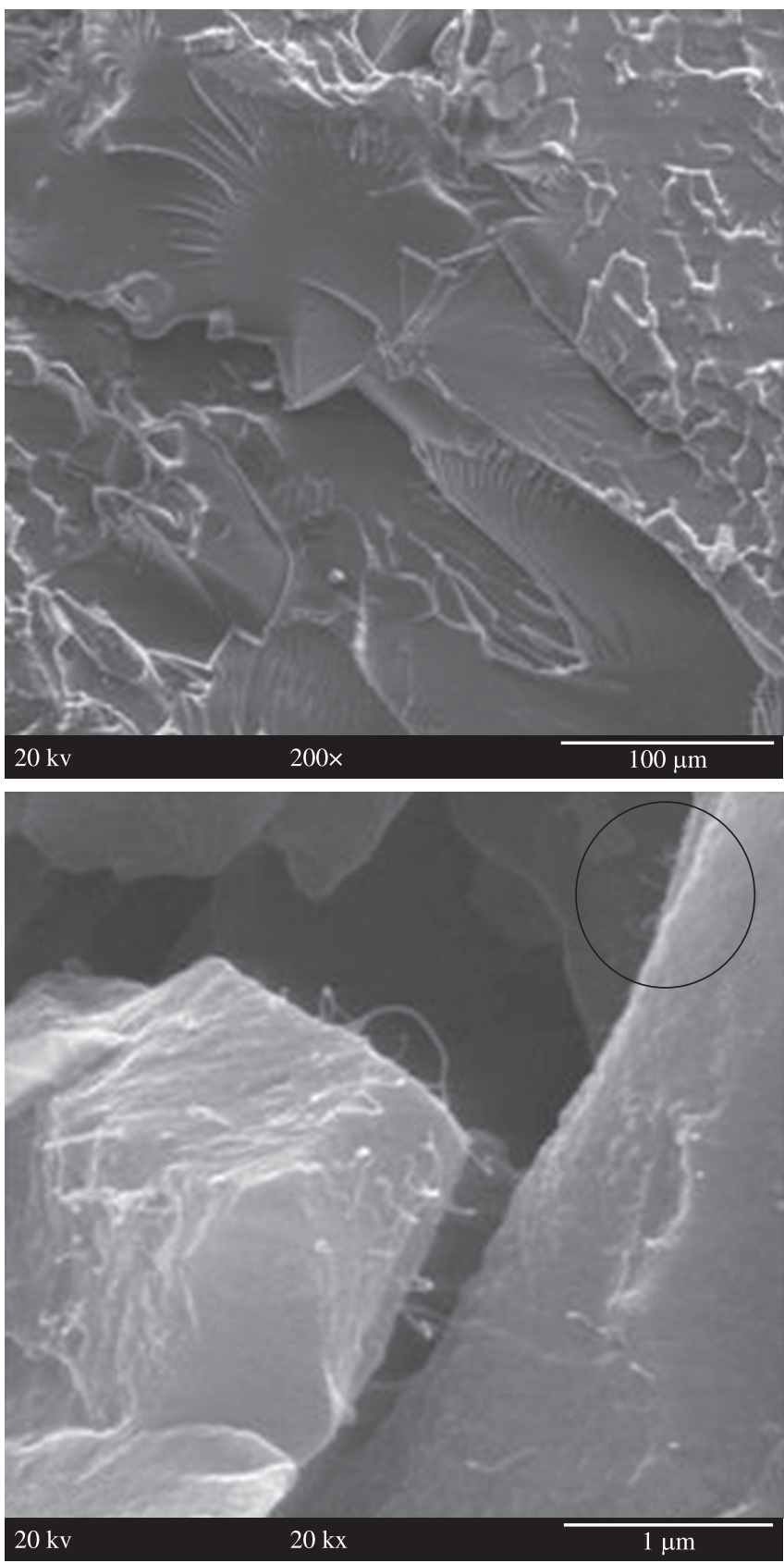

Figure 7. Scanning electron micrographs of the 0.25 wt. (\%) SWCNT-c/epoxy

\begin{tabular}{|c|c|c|c|c|c|}
\hline \multirow[t]{2}{*}{$\begin{array}{c}E_{\mathrm{f}} \\
(\mathrm{GPa})\end{array}$} & \multirow[t]{2}{*}{$L / D$} & \multirow[t]{2}{*}{$V_{\mathrm{f}}$} & \multicolumn{2}{|c|}{$\begin{array}{c}E_{\mathrm{c}}-\text { Rule of } \\
\text { Mixtures }(\mathrm{GPa})\end{array}$} & \multirow[t]{2}{*}{$\begin{array}{c}E_{\mathrm{c}}-\text { Halpin-Tsai } \\
(\mathrm{GPa})\end{array}$} \\
\hline & & & $\beta=0.20$ & $\beta=0.35$ & \\
\hline 500 & 500 & 0.0025 & 3.7 & 3.9 & 4.0 \\
\hline 500 & 1000 & 0.0025 & 3.7 & 3.9 & 4.0 \\
\hline 500 & 500 & 0.0050 & 4.0 & 4.4 & 4.4 \\
\hline 500 & 1000 & 0.0050 & 4.0 & 4.4 & 4.4 \\
\hline 1000 & 500 & 0.0025 & 4.0 & 4.4 & 4.2 \\
\hline 1000 & 1000 & 0.0025 & 4.0 & 4.4 & 4.3 \\
\hline 1000 & 500 & 0.0050 & 4.4 & 5.2 & 5.0 \\
\hline 1000 & 1000 & 0.0050 & 4.5 & 5.3 & 5.2 \\
\hline
\end{tabular}
nanocomposite prepared under mild sonication conditions.

Table 5. Predicted (theoretical) Young's modulus of the composites. 


\section{Conclusions}

In this work, nanocomposites were prepared by first dispersing carbon nanotubes, carboxylated or not, in the hardener. Different dispersion techniques were investigated, including mechanical/ manual stirring, use of solvent, use of surfactant and sonication (under distinct conditions). None of the variations significantly affected the final degree of curing of the nanocomposites, since no peak related to post-curing was observed in the first-run of the DSC analyses and also because the FT-IR yielded similar curves in the $910-920 \mathrm{~cm}^{-1}$ absorption range for neat and filled epoxy resins after curing.

The highest increase in glass transition temperature $\left(8^{\circ} \mathrm{C}\right)$ was obtained for a nanocomposite prepared with surfactant, solvent and under longer sonication at higher power. All nanocomposites presented higher thermal dilation than neat epoxy, even though the literature suggests that even small amounts of these nanofillers should decrease dilation. The storage modulus increased for all nanocomposites up to c.a. $65^{\circ} \mathrm{C}$, being c.a. $20 \%$ the highest increase, and the use of surfactant or solvent was not found imperative in influencing this property. Nanocomposites prepared with SWCNT-c presented higher microhardness than those prepared with SWCNT, except for SWCNT_C50_T40_A30_Mec, and it appears that solvent, time and power of sonication affect this property.

In general, tensile modulus increased with the addition of nanotubes to the epoxy but a less significant increase was found for tensile strength, which indicates poor CNTs/matrix adhesion even for the functionalized carboxilated CNTs studied. For both properties, higher values were found for nanocomposites prepared with a combination of solvent and surfactant. Flexural testing, on the other hand, indicated an increase in strength for some of the studied samples, whereas other samples showed similar strength and modulus in comparison with the neat resin.

SEM micrographs showed pull-out of carbon nanotubes in tensile tested specimens and suggested a reasonably homogeneous dispersion of the nanofillers, with an approximate distance of 100-200 $\mathrm{nm}$. Two simple theoretical micromechanical models were used to predict the expected range of Young's modulus of the nanocomposites and the calculated values were found to agree with the experimental results for most of the studied nanocomposites.

\section{Acknowledgements}

Part of this work was supported by PROCAD/CAPES, UNIESPAÇO/AEB and CNPq. In addition, one of the authors (L.A.F. Coelho) would like to thank Professor Vicente Amigó Borrás for his stay at UPV. We thank Prof. R. L. Moreira (UFMG) for his assistance in the infrared measurements, and Prof. Rodrigo Gibrel Lacerda (UFMG) for helpful discussions, suggestions and assistance.

\section{References}

1. Dyke CA and Tour JM. Covalent functionalization of single-walled carbon nanotubes for materials applications. Journal of Physical Chemistry A. 2004; 108(51):11151-11159. http://dx.doi.org/10.1021/jp046274g

2. Gojny FH and Schulte K. Functionalisation effect on the thermomechanical behaviour of multi-wall carbon nanotube/epoxy composites. Composites Science and Technology. 2004; 64(15):2303-2308. http://dx.doi.org/10.1016/j.compscitech.2004.01.024

3. Chen HY, Jacobs O, Wu W, Rudiger G and Schadel B. Effect of dispersion method on tribological properties of carbon nanotube reinforced epoxy resin composites. Polymer Testing. 2007; 26(3):351-360. http://dx.doi. org/10.1016/j.polymertesting.2006.11.004

4. Pizzutto CE, Suave J, Bertholdi J, Pezzin SH, Coelho LAF and Amico SC. Mechanical and dilatometric properties of carboxylated SWCNT/epoxy composites: Effects of the dispersion in the resin and in the hardener.
Journal of Reinforced Plastics and Composites. 2010; 29(4):524-530. http://dx.doi.org/10.1177/0731684408099924

5. Frankland SJV, Caglar A, Brenner DW and Griebel M. Molecular simulation of the influence of chemical cross-links on the shear strength of carbon nanotube-polymer interfaces. Journal of Physical Chemistry B. 2002; 106(12):3046-3048. http://dx.doi.org/10.1021/jp015591+

6. Prado LASD, De La Vega A, Sumfleth J and Schulte K. Noncovalent functionalization of multiwalled and double-walled carbon nanotubes: Positive effect of the filler functionalization on high glass transition temperature epoxy resins. Journal of Polymer Science Part B-Polymer Physics. 2009; 47(19):1860-1869. http://dx.doi.org/10.1002/polb.21789

7. Zhu J, Peng HQ, Rodriguez-Macias F, Margrave JL, Khabashesku VN, Imam AM et al. Reinforcing epoxy polymer composites through covalent integration of functionalized nanotubes. Advanced Functional Materials. 2004; 14(7):643-648. http://dx.doi.org/10.1002/adfm.200305162

8. Sumfleth J, Prehn K, Wichmann MHG, Wedekind S and Schulte K. A comparative study of the electrical and mechanical properties of epoxy nanocomposites reinforced by CVD- and arc-grown multi-wall carbon nanotubes. Composites Science and Technology. 2010; 70(1):173-180. http://dx.doi.org/10.1016/j.compscitech.2009.10.007

9. Suave J, Coelho LAF, Amico SC and Pezzin SH. Effect of sonication on thermo-mechanical properties of epoxy nanocomposites with carboxylated-SWNT. Materials Science and Engineering A-Structural Materials Properties Microstructure and Processing. 2009; 509(1-2):57-62. http://dx.doi.org/10.1016/j.msea.2009.01.036

10. Moniruzzaman M and Winey KI. Polymer nanocomposites containing carbon nanotubes. Macromolecules. 2006; 39(16):5194-5205. http://dx.doi.org/10.1021/ma060733p

11. Lau KT, Lu M, Lam CK, Cheung HY, Sheng FL and Li HL. Thermal and mechanical properties of single-walled carbon nanotube bundlereinforced epoxy nanocomposites: the role of solvent for nanotube dispersion. Composites Science and Technology. 2005; 65(5):719-725. http://dx.doi.org/10.1016/j.compscitech.2004.10.005

12. Hong SG and Wu CS. DSC and FTIR analysis of the curing behaviors of epoxy/DICY/solvent open systems. Termochimica Acta. 1998; 316(2):167-175. http://dx.doi.org/10.1016/S0040-6031(98)00356-6

13. Gauthier C, Chazeau L, Prasse T and Cavaille JY. Reinforcement effects of vapour grown carbon nanofibres as fillers in rubbery matrices. Composites Science and Technology. 2005; 65(2):335-343. http://dx.doi.org/10.1016/j. compscitech.2004.08.003

14. Loos MR, Coelho LAF, Pezzin SH and Amico SC. Effect of carbon nanotubes addition on the mechanical and thermal properties of epoxy matrices. Materials Research. 2008; 11(3):347-352. http://dx.doi. org/10.1590/S1516-14392008000300019

15. Loos MR, Coelho LAF, Pezzin SH and Amico SC. The effect of acetone addition on the properties of epoxy. Polímeros. 2008; 18(1):76-80.

16. Chen J, Hamon MA, Hu H, Chen Y, Rao AM, Eklund PC et al. Solution properties of single-walled carbon nanotubes. Science. 1998; 282(5386): 95-98. PMid:9756485. http://dx.doi.org/10.1126/science.282.5386.95

17. Zhang J, Zou H, Qing Q, Yang Y, Qingwen L, Zhongfan L et al. Effect of chemical oxidation on the structure of single walled carbon nanotubes. Journal of Physical Chemistry B. 2003; 107(16):3712-3718. http://dx.doi. org/10.1021/jp027500u

18. Gonnet P. Thermal conductivity and coefficients of thermal expansion of swnts/epoxy nanocomposites. [Dissertation]. Tallahassee: Florida State University; 2004.

19. Liao YH, Marietta-Tondin O, Liang ZY, Zhang C and Wang B. Investigation of the dispersion process of SWNTs/SC-15 epoxy resin nanocomposites. Materials Science and Engineering A: Structural Materials Properties Microstructure and Processing. 2004; 385(1-2):175-181.

20. Lau KT, Lu M and Liao K. Improved mechanical properties of coiled carbon nanotubes reinforced epoxy nanocomposites. Composites Part A: Applied Science and Manufacturing. 2006; 37:1837-1840. http://dx.doi. org/10.1016/j.compositesa.2005.09.019 
21. Wichmann MHG, Schulte K and Wagner HD. On nanocomposite toughness. Composites Science and Technology. 2008; 68(1):329-331. http://dx.doi.org/10.1016/j.compscitech.2007.06.027

22. Coleman JN, Khan U, Blau WJ and Gun'ko YK. Small but strong: A review of the mechanical properties of carbon nanotube-polymer composites. Carbon. 2006; 44(9):1624-1652. http://dx.doi.org/10.1016/j. carbon.2006.02.038
23. Gojny FH, Wichmann MHG, Kopke U, Fiedler B and Schulte K. Carbon nanotube-reinforced epoxy-composites: Enhanced stiffness and fracture toughness at low nanotube content. Composites Science and Technology. 2004; 64(15):2363-2371. http://dx.doi.org/10.1016/j. compscitech.2004.04.002

24. Liu LQ, Tasis D, Prato M and Wagner HD. Tensile mechanics of electrospun multiwalled nanotube/poly(methyl methacrylate) nanofibers. Advanced Materials. 2007; 19(9):1228-1233. http://dx.doi.org/10.1002/ adma.200602226 